\title{
RELACIÓN DE CUIDADO MUJER/CRIANCA: DOMINACIÓN SIMBÓLICA Y DESIGUALDAD
}

\author{
WOMAN/CHILDREN CARE RELATIONSHIP: SYMBOLIC DOMINATION AND INEQUALITY
}

RELAÇÃO DE CUIDADO MULHER/CRIANÇA: DOMINAÇÃO SIMBÓLICA E DESIGUALDADE

\section{RESUMEN}

El objetivo del presente artículo fue analizar las representaciones de género que circulan en la relación de cuidado mujer/criança, a partir del análisis de los datos obtenidos en el trabajo de campo de corte cualitativo, desarrollado en el barrio Potosí de la ciudad de Bogotá. En la que se halló que dicha relación de cuidado se encuentra en un escenario de tensiones, ya que las prácticas de cuidado son accionadas por las vulnerabilidades presentes en el territorio, convergen diversas formas de opresión. Entre ellas, la atribución naturalizada del cuidado de la primera infancia a las mujeres y la tendencia a caracterizar el niño como un ser fuerte y activo, mientras la niña es caracterizada por su delicadeza y debilidad. Lo cual, corresponde a un sistema invisible de disposiciones, manteniendo el orden social y como consecuencia estructurando relaciones sociales que reproducen dinámicas de desigualdad.

Palabras clave: Cuidado. Género. Mujer y Niñez.

\begin{abstract}
The objective of this article was to analyze the representations of gender around the relationship of care woman/children, from the qualitative analysis of the data obtained in the field work developed in the neighborhood called Potosi in the city of Bogotá. The finding shows that this care relationship is in a scenario of tensions, since the practice of the care is triggered by the vulnerabilities present in the territory converging in various forms of oppression. Among them, the naturalized attribution of early childhood care to women and the tendency to characterize the boy as strong and active, while the girl is characterized by her delicacy and weakness. This corresponds to an invisible system of provisions, maintaining social order and as consequence, structuring social relations that reproduce inequality and vulnerability dynamics.
\end{abstract}

Keywords: Care. Gender. Woman and Childhood.

\section{Yeimy Carolina Espitia Villafañe}

Másteren Economía Doméstica da Universidade Federal deViçosa (PPGED-UFV), MG, Brasil. Licenciada em psicologia e pedagogia da Universidade Pedagógica Nacional. E-mail: yeimyespitiavillafane@gmail. com. orcid.org/oooo-0002-0745-9872

Rita de Cássia Pereira Farias

Doctora en Antropología da Unicamp. Professora e investigadora da Universidade Federal de Viçosa, MG, Brasil. E-mail: rcfarias@ufv.br. orcid.org/oooo-0oo2-8108-5370 


\section{RESUMO}

O objetivo deste artigo é analisar as representações de gênero que circulam na relação mulher/criança ${ }^{1}$, a partir da análise qualitativa dos dados obtidos na pesquisa de campo desenvolvida no bairro Potosí, na cidade de Bogotá, Colômbia. Constatou-se que a relação de cuidado se dá em um cenário de tensão, visto que as práticas de cuidado são desencadeadas pelas vulnerabilidades presentes no território, convergindo em diversas formas de opressão. Entre eles, a naturalização da atribuição de cuidados na primeira infância às mulheres e a tendência a caracterizar o menino como um ser forte e ativo, enquanto a menina se caracteriza por sua delicadeza e franqueza, o que corresponde a um sistema invisível de disposições que mantém a ordem social e estrutura as relações sociais que reproduzem dinâmicas de desigualdade.

Palavras-chave: Cuidado. Gênero. Mulher e Infância.

\section{Introducción}

El trabajo de cuidado se refiere a una serie de actividades que responden a necesidades concretas de bienestar, este no puede ser entendido sin reconocer que tiene una carga subjetiva, una posición moral. Lo que remite a comprender la ética de cuidado, la cual está asociada a la responsabilidad en términos relacionales, significa que es en el hecho de relacionarse con el otro lo crea la responsabilidad (TRONTO, 1987; MOLINIER; PAPERMAN, 2015). Por lo tanto, para no contemplar sólo a las personas que cuidan familiares en casa o las que lo realizan como trabajo remunerado, se propone entender esta actividad como una red de responsabilidad, como parte de la sociedad, porque la sociedad necesita de care ${ }^{2}$ (HIRATA, 2010).

El trabajo de cuidado ya sea este remunerado o no, es realizado mayoritariamente por las mujeres. Son ellas quienes invierten tiempo, fuerza física y mental en la ejecución de la limpieza, la cocina, la organización, entre otras labores, a favor del bienestar de otro. Aunque no son todas las mujeres quienes realizan esta labor sino especialmente las que ocupan una posición de subalternidad (MOLINIER, 2012).

Ello se evidencia fuertemente en lo que respecta la asignación del cuidado de la niñez en la ciudad de Bogotá, territorio en el que se desarrolló la presente investigación. Según las cifras, el cuidado de la primera infancia está a cargo de las mujeres, pues el 36\% de las mujeres que trabajan o deben salir del hogar dejan sus

\footnotetext{
1 Si bien, podría hablar de la relación madre/hija-hijo, se habla de mujer/criança porque se quiere posicionar el ser mujer ante del hecho "naturalizado" de ser madres y además porque no solo las madres realizan la labor de cuidado en la comunidad investigada, pues abuelas, tías, hermanas, etc., también hacen parte de esta actividad. Por otra parte, se tomó la palabra criança del idioma portugués, porque se encuentra que es una palabra neutra para hablar del sujeto denominado niño o niña en el español.

2 "O termo care é dificilmente traduzível, porque é polissêmico. Cuidado, solicitude, preocupação com o outro, estar atento a suas necessidades, todos esses diferentes significados estão presentes na definição do care" (HIRATA, 2010, p. 42)
} 
hijas e hijos al cuidado de las abuelas, el 25\% los lleva al lugar de trabajo, el 9\% los deja con su cónyuge, el $8 \%$ buscan ayuda de otros parientes, el 5\% de la empleada doméstica, $4 \%$ de los vecinas, $3 \%$ de la niña mayor y tan sólo el $4 \%$ recurre al cuidado que el Estado ofrece a través del instituto colombiano de bienestar familiar (ICBF) (BOGOTÁ, 2014). Aspecto esencial para que esta investigación se centre en el ámbito doméstico y específicamente en el trabajo de cuidado con primera infancia.

Así que, la presente investigación trabaja sobre el aspecto del cuidado en el ámbito familiar, explícitamente en la relación mujer/criança, a partir del siguiente cuestionamiento ¿las representaciones de género que circulan en la relación de cuidado mujer/criança presentan prácticas de dominación simbólica?

De esta manera, en la primera parte del texto se describe la forma en que se establece la relación de cuidado mujer/criança, dado que el cuidado de la niñez ha sido entendido como una responsabilidad tradicional de las mujeres. Este esquema hace parte de la división sexual y social del trabajo, pues, continúa siendo evidente la clara separación y jerarquización entre los trabajos masculinos y femeninos, aunque no en todas las sociedades se presente de una única forma (HIRATA; KERGOAT, 2007). Además de ser una de las desigualdades más persistentes en la sociedad, en la que la presencia de hijos o hijas representa una situación más angustiante para las mujeres, que para los hombres (HIRATA, 2015).

Por consiguiente, se comprende el género como elemento constitutivo de las relaciones sociales basadas en la diferencia entre lo que es definido como masculino y femenino, es una forma primaria de dar significado a las relaciones de poder, que si bien, no es el único campo en el que se encuentra el poder como expone Scott (1996), parece ser una forma persistente y recurrente de facilitar la significación de poder, como se presentará en la segunda parte del texto.

En esa medida las representaciones de género comprendidas como constitutivas de las relaciones sociales permiten entender cómo las crianças son cuidadas de formas diversas, basadas en las diferencias sociales que distinguen los sexos. Lo cual hace parte de unas disposiciones socialmente constituidas, elemento que se expone en la última parte del texto al describir las prácticas de dominación y desigualdad que se presentan en la relación de cuidado mujer/ criança.

\section{Metodología}

Esta investigación se caracteriza por ser de corte cualitativo, en la que se comprendieron las investigadas como partícipes del estudio, porque su accionar fue la base del trabajo investigativo (FALS BORDA, 2009). Para su desarrollo se tomó una muestra intencional en el barrio Potosí, el cual está ubicado al sur de la ciudad Bogotá. Esta zona se caracteriza por ser conflictiva, por tener una alta tasa de fecundidad y de embarazos adolescentes, además la población de los niños y las niñas está por 
encima del promedio distrital y aproximadamente el 56\% de las mujeres no participa del mercado laboral (EDDS, 2011).

La muestra se conformó por 18 mujeres (entre 16 y 65 años) que cuidan de las crianças en la primera infancia, sin ser remuneradas por dicha actividad. Las mujeres aceptaron participar de la investigación después de leer y firmar el término de conocimiento y libre esclarecimiento (TCLE), atendiendo a la Legislación Brasileña, en especial la Resolución 466/2012 Del Consejo Nacional De Salud.

Se realizaron 18 entrevistas semiestructuradas y luego dos entrevistas de grupo focal en las que participaron 8 mujeres. Los datos obtenidos se comprenden dentro del esquema propuesto por Bourdieu (2007), o sea, se percibió lo social como una realidad histórica, constituida por campos, microcosmos o espacio de relación objetiva que tienen una lógica propia, en el que se encuentra por una parte el campo de fuerzas donde el sujeto es oprimido y por otra el campo de luchas, en que los agentes actúan de acuerdo a sus posiciones relativas en el campo de fuerzas conservando o transformando la estructura (THIRY-CHERQUES, 2006).

En esa medida, en la investigación los hechos sociales se complementaron con tendencias que aparecieron como actos sucesivos válidos en los contextos inmediatos que no pueden ser comprendidos sino se adentra en la historia (FALS BORDA, 2009). Ello permitió dar cuenta de las prácticas presentes en la relación de cuidado estudiada. La idea fue propiciar un intercambio entre conceptos y hechos sociales, ubicando las tendencias que se observaban en la realidad.

\section{Relación De Cuidado: Mujer/Criança}

El trabajo de cuidado es realizado mayoritariamente por mujeres (HIRATA, 2015; MOLINIER, 2012) y ello se evidencia especialmente en lo que respecta la asignación del cuidado de la niñez, aspecto sobre el que se ha centrado este texto, debido a la serie de tensiones bajo las que se configura dicha atribución.

Según este recorte investigativo, se presente como tendencia ${ }^{3}$ naturalizar el cuidado de la niñez como responsabilidad de la mujer, o sea, es visto como un deber, encasillado en lo biológico y asociando el cuidado a labor de la maternidad ${ }^{4}$ (QUIROGA, 2014). Disposición socialmente interiorizada que refleja la objetivación de la estructura,

3 Tendencias: actos sucesivos válidos en los contextos inmediatos que se observaban en la realidad. La teoría se construye por medio de preconceptos, ideas preliminares o informaciones, cosas en sí, hecho y práctica (FALS BORDA, 2009).

4 En este apartado se discute sobre la maternidad como estereotipo unificador de las mujeres, que rechaza las individuales y elementos tales como la clase social o nivel cultural, siendo parte de las representaciones dominantes de la maternidad que "se desarrollan en relación con construcciones políticas y sociales construidas a su alrededor y mantenidas por el sistema de género que les subyace" (SALETTI, 2008, p. 177). 
La mamá es lo primordial, porque uno de mamá sabe cómo cuidar sus hijos, porque uno los tuvo nueve meses en el vientre, entonces uno sabe cómo es ese bebé, uno sabe que le duele, uno tiene la idea de que le molesta, si tiene calor, si tiene frío, si tiene hambre, si necesita algo, si esta aburrido, uno de mamá sabe eso (Natalia, 37 años).

En consecuencia, la responsabilidad tradicional de cuidado de la niñez por parte de las mujeres no ha presentado muchos cambios, ya que parece que la presencia de hijos o hijas, según Hirata (2015), representa una situación aún más angustiante para las mujeres que para los hombres, como señalan las mujeres entrevistadas,

Ingresar a mi hijo al jardín lo pienso mucho, por muchas cosas que han pasado, el abuso a los niños, que me lo maltraten $¿$ si me entiendes? eso me da mucho miedo. Entonces, [es mejor] buscar una forma de trabajo como estoy haciendo ahorita, vender yogurt, yo misma lo preparo; salir, ofrecerlos, estoy pendiente de ellos [mis hijos] y pues no los descuido tampoco (Lina, 28 años).

Relato que ilustra situaciones prácticas que mantienen el cuidado en la esfera privada, al ser moldeada por acciones invisibles, es decir, por la estructura de mercados, las relaciones sociales, los tiempos, los espacios, en últimas en todos los ámbitos de la vida se generan tensiones para que esta práctica se conserve en dicho espacio; por ejemplo, en lo que respecta el mercado laboral las mujeres señalan,

Uno se pone a pensar, yo trabajo porque lo necesito. Pero usted va y recibe un mínimo [cada mes], que la señora que lo cuide [al niño] le cobra 150 [cada mes], más lo que tiene que dejar de medias nuevas o de almuerzo digamos 100 más, son 250 y usted tiene que gastar en sus pasajes otros 150 al mes, son 300 y los otros 300 [solo] son para ayudar en la casa, entonces usted hace más en la casa, cuida a su hijo y se ahorra esos 300 (Leticia, 33 años).

Los anteriores diálogos evidencian la estructura del poder desigual en el mercado económico, que se refleja en el campo familiar (HIRATA, 2015; QUIROGA, 2014), lo que es un principio generador de posiciones, dado que coloca a las mujeres en lugares de desigualdad. Porque al ser ellas quienes deben realizar el trabajo de cuidado quedan económicamente dependientes de su pareja u otras personas (hijos, nueros, etc.) o deben realizar trabajos remunerados sin garantías.

Por consiguiente, existe una conexión entre el posicionamiento de la mujer que realiza el trabajo doméstico no pago y su permanencia en la unidad familiar con el hecho de cuidar las niñas y los niños en la primera infancia, lo que se ha caracterizado en este artículo como la relación de cuidado mujer/criança. 
Tendencia que acorta la red de responsabilidad de esta práctica de cuidado, ya que, las mujeres comprenden esta labor como un deber solo de la mujer/madre. Esa posición hace parte de las dinámicas de dominación simbólica de acuerdo con Bourdieu (200o), que son el reflejo de una división sexual y social del trabajo, o sea, de la clara separación y jerarquización entre los trabajos masculinos y femeninos (HIRATA, 2015).

En esa medida y en concordancia con Moliner (2012) no es posible comprender los oficios domésticos aparte del trabajo de cuidado, aspecto que permite profundizar sobre cómo se establece la relación de cuidado aquí descrita. Ya que, en las conversaciones de las mujeres sobre su cotidianidad en lo que se refieren a los quehaceres domésticos, se demuestra una relación directa entre la ejecución de actividades domésticas y el trabajo de cuidado. Lo que ha conllevado a que la atribución del trabajo doméstico a las mujeres permanezca intacta en todas las regiones del mundo -con algunas diferencias-, ya que, esta esfera se encuentra permeada por la ideología patriarcal, manteniendo estereotipos y modelos (HIRATA, 2015).

Esta lectura permite reconocer algunos aspectos de cómo la sociedad está organizada alrededor del cuidado de la niñez, lo que moldea y orienta las relaciones de cuidado en el territorio. Porque, al ubicar la posición de esta relación se comprende como una de las razones que mantiene la separación y jerarquización entre los trabajos masculinos y femeninos, dado que se naturaliza el cuidado de las crianças como responsabilidad de la mujer y consecuentemente son acortas las redes de responsabilidad, conservando la estructura de división sexual y social del trabajo.

Es en este escenario de tensiones en el que se establece la relación del cuidado mujer/criança, por tanto es necesario ahondar sobre las construcciones sociales que circulan y hacen parte de esta relación, ya que como indica Bourdieu (2007) las acciones parten de la construcción de mundo que tienen los sujetos y son manifestaciones del poder simbólico, lo cual se abordará en el siguiente seccion.

\section{Género Y Cuidado De La Primera Infancia}

El cuidado se refiere a una serie de actividades que responden a necesidades concretas de bienestar y experiencias materialmente estructuradas (MOLINER, 2015), y que es realizado por un agente ubicado en un campo de fuerzas a partir de unas posiciones construidas en el mundo social, como se expuso en el anterior apartado, lo que lleva a cuestionar sobre ¿cuáles son los habitus ${ }^{5}$ que circulan en el cuidado de la primera infancia?

5 Os habitus são princípios geradores de práticas distintas e distintivas [...] mas são também esquemas classificatórios, princípios de classificação, princípios de visão e de divisão e gostos diferentes (BOURDIEU, 2007, p. 22). 
Subrayar antes que distintas escuelas del psicoanálisis se han interesado por cómo se crea la identidad del sujeto, centrándose en las primeras etapas del desarrollo de la niñez (SCOTT, 1996). La teoría de las relaciones objetales, resalta que el cuidado de la infancia típicamente ha sido sinónimo del ejercicio individual de la maternidad y por ende la relación temprana es con una madre (CHODOROW, 1978), lo que permite deducir que el desarrollo temprano consiste en la construcción de una relación social y emocional entre la madre y el bebé, y esto tanto en el mundo como dentro de la psique infantil.

Sin embargo, ninguna investigación demuestra que existe la necesidad que una única persona cuide del infante, pues varios adultos y crianças pueden estar más próximo a la norma histórica y cultural, que la que actualmente se ha llegado a considerar "natural" (madre/criança) (CHODOROW, 1978). A ello se suma que las camadas pobres se caracterizan por las redes de solidaridad intergeneracional, sistema de cuidados basado en la familia extensa (SORJ; FONTES, 2012), como, por ejemplo, la entrevistada Luna (62 años) cuida de 3 nietos/as y 2 bisnietos/as.

Ahora bien, comprendiendo los habitus como generadores de prácticas y representaciones distintas y distintivas (BOURDIEU, 2007), se podría señalar en un primer momento que en la relación de cuidado mujer/criança no se presentan prácticas distintivas de cuidado, según la muestra de esta investigación ¿Acaso un niño es diferente a una niña?" (Mariana, 19 años), pues se está hablando de "un ser inocente, o sea, que toca cuidarlo, que toca quererlo, que toca respetarlo, eso es ser un niño, enseñarlo a jugar [...]" (Natalia, 37 años). En este sentido, esa relación parece contener una perspectiva de igualdad. Empero, durante los diálogos de las entrevistas, dicha visión va cambiando, a medida que se profundiza sobre las representaciones simbólicas que hacen parte de las prácticas de cuidado, apareciendo en primer momento un distintivo, el cuerpo:

Niño y niña son iguales [cuando uno los cuida], [...] lo único diferente que tienen es el sexo, o sea que el niño tiene su cosito y la niña tiene su vagina, eso es lo único diferente de resto son niños que juegan, se divierten (Laura, 20 años).

Surge poco a poco visiones y divisiones del universo social que recaen sobre el hecho de cuidar de una criança, lo que comienza a partir de la elaboración social de sexo y cuerpo, presentando tensiones y conflictos de como el cuerpo se relaciona con las identidades masculina y femenina (SCOTT, 1996).

En los diálogos de las mujeres partícipes de la investigación se hacen evidentes unos símbolos culturales sobre lo que es una niña, colocándose en oposición al significado de niño o viceversa, inclusive desde antes de nacer; “[...] los niños desde el vientre se mueven más, piden hasta más de comer, mientras las niñas de por sí, yo lo digo por mis niñas, son dóciles" (Inés, 28 años). Esos sujetos son inseridos en unos campos que corresponden a construcciones sociales, a través de unos esquemas de percepción tanto de pensamiento como de acción, 
Para mí un niño es como más fuerte, o yo no sé. La verdad las niñas las he visto muy frágiles, muy tiernas. El niño se me hace que es más fuerte, como más ordinario, como que ellos hacen algo y esto, todo a la brusquedad (Paola, 19 años).

Lo que permite señalar, que en el universo social de las mujeres entrevistadas se encuentra una tendencia en los que respecta la representación del sujeto denominado niña, al ser caracterizada por su fragilidad y delicadeza, ya que al niño se le caracteriza por su fuerza y energía, división que aparece naturalizada bajo esquemas cognitivos.

Así pues, en concordancia con Bourdieu $(1998 ; 2000)$ se puede decir que la experiencia en el mundo social comienza por una división socialmente construida entre los sexos, que se legitima y naturaliza en una realidad colectiva. Existe un trabajo de construcción práctico que impone una definición diferenciada en las crianças, que en este caso inicia con la forma en que son cuidadas.

$\mathrm{Si}$ bien parece que el cuidado de la primera infancia es unificado, este se ejerce con el imaginario de que "ella es la niña, ella es la femenina, él es el más fuertecito, a que no sean los niños tan sensibles como las niñas" (María, 26 años). Tales características establecen lazos que permiten la trasmisión de rituales, que son incorporados en el colectivo para que se reproduzcan socialmente una variedad de prácticas (BOURDIEU, 1999). Lo que demuestra cómo recaen sobre las crianças la historia que ha construido y determinado a los sujetos, o sea, en últimas de lo que se está hablando es de un proceso de construcción del sujeto (HARAWAY, 1995).

Eso permite resaltar otra tendencia en la relación de cuidado, la cual responde a prácticas de construcción de identidad, las niñas - caracterizadas por la fragilidad y delicadeza - son cuidadas para protegerlas de dinámicas sociales, es decir, se hace hincapié en las violencias a las que está expuesta la mujer, "toca cuidar mucho a las niñas, pues tantos peligros que hay" (Luna, 62 años).

Por otra parte, el niño es cuidado al ser disciplinado, debido a la visión de que este es más activo; como lo menciona Leticia: "mi hijo es terrible, el jode y a él ha tocado sufrir por parte mía que es lo peor, [...] porque yo soy dura con él”.

Este es un trabajo de construcción práctica que orienta y estructura las representaciones, pues la expresión "ser más dura” con la criança que representa el niño, impone prácticas diferenciadas, ya que con la niña predomina la protección. Lo que desencadena disposiciones en la actividad de cuidado, correspondientes a dinámicas de poder simbólico, como producto de un aprendizaje social. De esta forma, en la estructura mental son moldeadas las acciones de los niños y las niñas, para en últimas mantener la estructura de las relaciones sociales.

Así las prácticas de cuidado en dicha relación reflejan las representaciones de género, que estructuran la percepción y la organización, concreta y simbólica del mundo social (SCOTT, 1996). Como indica una de las entrevistadas, al ser movida en razón a una identidad que se define como masculina, las prácticas de cuidado acaban por reforzar determinados patrones dominantes de masculinidad: 
Yo creería que las que piensan que así pequeñitos, uno estarle poniendo que muñequitas [a los niños], que losita, que, a la cocina, entonces no se va [a formar], no es que sea machismo, es por identidad. Uno los confunde, se va criando esa mentalidad. [...] yo pienso que desde chiquitos si hay que inculcarles (Rosa, 35 años).

Este diálogo permite comprender cómo es representado el sujeto criança, según la muestra tomada, al ser una forma de denotar las "construcciones culturales", la creación social de ideas de las formas de ser niño o/y niña. Por tanto, las crianças son cuidadas de forma diferente basadas en las diferencias sociales (no biológicas) que distinguen los sexos (SCOTT, 1996), inclusive antes de nacer. Entonces, se desarrollan hábitos sistemáticamente diferenciados y diferenciadores, al interiorizar la división social de los dos sexos a partir de una construcción correspondiendo a un orden social,

María (26 años): [...] en la vida hay que diferenciar que es una niña y que es un niño, más allá de que tengan órganos sexuales diferentes.

Dennis (36 años): y más ahorita viendo que realmente hay mucho niño u hombres que se han confundido mucho, yo pienso no, de que sea ha confundido mucho y por eso estamos como estamos ahorita y si lo pone uno desde chiquiticos a jugar con lo de niñas, pues terrible.

Natalia (37 años): Crecen con esa mentalidad.

Inés (28 años): Cuando uno les compra las cosas a los niños es para que la gente los diferencie, porque que tal vestir a la niña siempre de azul o amarillo, la gente va a decir ja ella tuvo fue un niño! viendo que es niña, si es niña toca abrir los oídos para que se vea niña, si no le van a decir que tan lindo ese niño. (Entrevista de Grupo Focal, 2017).

Es de esta manera que distintos elementos de la estructura social, especialmente los que se circulan a través de la relación mujer/criança, son apropiados y transformados internamente y van a influir en la vida afectiva y la estructura psíquica de la criança, pues se apropia de las relaciones conflictivas tal como las experimenta, y organiza internamente estas experiencias en el self (CHODOROW, 1984).

En últimas, lo que permite esta mirada sobre las construcciones simbólicas y por ende prácticas que transitan en la relación de cuidado mujer/criança, es comprender como antes de nacer los sujetos son inseridos en la cultura y como los cuidados responden a las dinámicas impuestas en la sociedad.

Tales disposiciones permiten deducir que, como afirma Scott (1996) el género es constitutivo de las relaciones sociales, las cuales están basadas en diferencias que 
distinguen los sexos, estando presente representaciones simbólicas, siendo estas una forma de referirse a los orígenes exclusivamente sociales de las identidades subjetivas de hombres y mujeres. Además, dicha percepción de la realidad se funda en que las relaciones de género se organizan a partir de la oposición binaria que corresponde a la dominación simbólica, como se expondrá en el siguiente apartado, organizando dinámicas de igualdad y desigualdad en el mundo social.

\section{Dominación En La Relación De Cuidado Mujer/Criança}

Las prácticas de cuidado en la relación mujer/criança, parten de una visión y división del mundo, pues, según el recorte investigativo la niña es caracterizada por su fragilidad y delicadeza, el niño por su fuerza y energía, división y jerarquización que aparece naturalizada bajo esquemas cognitivos que son accionados por disposiciones socialmente construidas (BOURDIEU, 2000). Por ende, la niña es cuidada para ser protegida y el niño para ser disciplinado, respondiendo a las representaciones de género que circulan sobre las crianças, disposiciones entendidas como actos sucesivos que se hallaron en la investigación y que dan cuenta de unas tendencias de acción en esta actividad. Pero ¿qué genera estas prácticas de cuidado?

Se reconoce que la relación de cuidado mujer/criança, por una parte, se remite al desarrollo temprano y que este consiste en la construcción de una relación social y emocional entre una cuidadora (o/y varios/as cuidadores/as) y el bebé a favor de su supervivencia (CHODOROW, 1978). Por otra parte, se entiende que el care no debe ser interpretado solamente como afectivo y/o psicológico, pues el sujeto que lo realiza actúa y piensa en función de unas experiencias materialmente estructuradas (MOLINER, 2012) porque conoce las múltiples formas de vulnerabilidad del espacio en el que se encuentra, proceso en el que se destaca el acto de relacionarse como unidad de análisis (MOLINIER; PAPERMAN, 2015).

Sumado a lo anterior, se entiende que el relacionar pasa por la forma de entender las relaciones sociales y sexuales, según Scott (1996), por tanto, las prácticas de cuidado corresponden a unas dinámicas de relación social. Así que, la relación de cuidado se encuentra una doble imposición objetiva y subjetiva (BOURDIEU, 2006). De esta forma, para comprender dichas prácticas de cuidado, se debe abarcar tanto los sujetos como la organización social (SCOTT, 1996).

Resaltando, las prácticas de cuidado son accionadas por una estructura social, o sea, dichas acciones por más personales y transparentes, no pertenecen al sujeto en su totalidad, sino al sistema completo de relaciones en las cuales y por las cuales ellas se realizan (THIRY-CHERQUES, 2006). Esto se puede analizar con una frase que expresan las personas cuando saben de un embarazo: "ojalá sea niño, porque las niñas sufren más” (Entrevista de grupo focal, 2017) y que aparece en repetidas ocasiones en el trabajo de campo, siendo un detonante de cómo entender lo "socialmente construido", en lo que concierne a cómo cuidar de la primera infancia. 
Pues, si bien la frase da cuenta efectivamente de una estructura que persiste en las relaciones sociales, que se basa en diferencias que distinguen los sexos (SCOTT, 1996), como se señaló. También, esta frase remite a reflexionar sobre como la división muestra posiciones de dominación que mantienen el orden social, es decir, existen unas dinámicas estructurales que conservan dichas relaciones, lo que se evidenció en una las entrevistas de grupo focal:

Investigadora: ¿Por qué las niñas sufren más que los niños? Ina (16 años): Yo quisiera tener una niña, pero a veces digo no porque veo lo que muchas veces les pasan a las mujeres, a veces digo que no más [me quedo] con él [mi hijo/hombre]. Hasta él [mi esposo] piensa que una mujer sufre más.

Natalia (37 años): Una mujer lleva todo, hoy en día nadie respeta a las mujeres. No sé si vieron la noticia de ese hombre que le pego a esa mujer 25 palazos, en eso también me baso hoy en día.

Ina (16 años): Hasta los papás, hasta los propios tíos, hasta los abuelos.

Mariana (19 años): El papá que violó a la hija de nueve años y más encima la mató.

Leticia (33 años): El papá es más duro.

Ina (16 años): Eso es lo que uno piensa, porque a veces las niñas no cuentan y se guardan esas pequeñas cosas.

Leticia (33 años): Yo les explico a mi hija nadie te puede tocar la cola, mi hija me cuenta todo, si tu papá, si tu hermano, todo (Entrevista Grupo Focal, 2017).

El diálogo muestra que el cuidado de las niñas está asociado a la protección, tendencia que se expuso en el anterior apartado, pero también muestra cómo la representación de debilidad está relacionada con la vulnerabilidad, debido a los escenarios de violencia que las cuidadoras perciben en el universo social ${ }^{6}$, lo que lleva a deducir que la representación de la niña como ser sensible y frágil surge a partir de un orden social en el que la mujer es vulnerada,

Una niña se tiene que cuidar más, porque un niño uno lo cuida que digamos que de pronto un niño adolescente que no coja malos pasos. En cambio, una niña debe tener más cuidado, por tanta maldad, sé que también para los niños hay maldad, pero para las niñas hay más. Una maldad, a veces los humanos que piensan en violar, hacerle daño, [...] una niña debe tener más cuidado, aunque tiene que ser por igual, pero yo digo que uno debe cuidar más a una niña que a un niño (Karen, 18 años).

6 En Ciudad Bolívar, zona en la que se encuentra el barrio Potosí, el 46\% de las mujeres sufren violencia física por los cónyuges, además es una localidad caracterizada por la alta presencia de embarazos adolescentes (EDDS, 2011). 
Razón por la cual las mujeres tienen una visión de protección con la niña/mujer, destacando una jerarquía entre las niñas y los niños, debido a la naturalización de lo que se percibe como masculino y femenino, que es reforzado por la presencia de la violencia contra las niñas/mujeres. Sin embargo, ante estas prácticas de dominación, aparecen algunos matices de resistencia: "Ellas también tienen que aprender a ser fuertes, tanto afuera como aquí adentro de la casa [...]" (Ina, 16 años).

Las anteriores palabras exponen como las mujeres son conscientes del ejercicio que realizan, puesto que la "fuerza" permite responder a las dinámicas sociales. Atendiendo a ello, el ejercicio de disciplinar a los niños/hombres equivale al temor de estos "coger malos pasos" (Karen, 18 años). Según las mujeres este es más propenso a ser problemático en el futuro y/o a ser parte de los grupos del territorio 7 si no es disciplinado. Estas prácticas permiten comprender el surgimiento de las representaciones simbólicas que circulan en el trabajo de cuidado con la primera infancia.

Así pues, lo que señalan las acciones de cuidado según la muestra, es que las representaciones simbólicas de niño y niña que son aprendidas socialmente y circulan en la relación de cuidado mujer/criança surgen a partir de los escenarios de vulnerabilidad que se encuentran en el territorio, como lo son la alta presencia de redes narcotráfico, violencia intrafamiliar, violencia a la mujer, entre otras.

Además, en los diálogos se expone claramente que las mujeres hacen parte del proceso en la que los niños y las niñas aprenden a comprender el mundo. Por ende, de acuerdo con Bourdieu (2007), por más fuerte que sea la estructura se encuentra un espacio de lucha cognitiva, porque ellas son parte de la estructura y por tanto pueden transformarla. Ello se puede evidenciar en la tendencia que muestra una necesidad de que los niños/hombres sean parte activa del mundo doméstico,

Este niño [mi hijo], no porque es niño, no quiere decir que no [va a hacer nada], yo ya lo pongo a que ayude a lavar loza, a que ayude a recoger reguero, a que le lleve las chanclas a las niñas, porque él no tiene aquí quien le haga, o sea él no tiene, porque nosotras hacerle nada, o sea, yo no pienso educar a eso (Dennis, 36 años).

Esta visión va colocando espacios de resistencia que surgen en el ejercicio de cuidar, porque las mujeres conciben, "en la definición de niña y niño la oportunidad de un futuro mejor" (Mary, 26 años).

En clave a esas experiencias las mujeres buscan generar bienestar a las crianças, en últimas son esas representaciones como diría Scott (1996) las que estructuran la percepción y la organización, concreta y simbólica del mundo social, al responder a las dinámicas de violencia que se encuentran en el territorio, modeladas por esas

7 Este territorio se caracteriza por alta tasa de adolescentes asesinados, "cada fin de semana se registran al menos tres asesinatos de jóvenes, entre los 14 y los 26 años en la localidad de Ciudad Bolívar. Solo en el primer semestre del año 2015 ocurrieron 125 homicidios en esta" (CHICA, 2015). 
estructuras de dominación que las producen (BOURDIEU, 20oo). Por tanto, la relación de cuidado de mujer/criança se establece en un escenario de vulnerabilidad y es muestra de las formas de opresión que ocurren en dicho territorio, lo que causa el mantenimiento de relaciones de dominación y de desigualdad en las familias y la sociedad, que se visibilizan en el sistema de disposiciones en el que se encuentran las mujeres y la primera infancia.

\section{Reflexiones Finales}

El presente artículo permite afirmar que existe una conexión entre el posicionamiento de la mujer que realiza el trabajo doméstico no pago y su permanencia en la unidad familiar con el hecho de cuidar a la primera infancia, lo que se caracterizó como la relación de cuidado mujer/criança.

Según la muestra investigativa puede comprenderse dicha relación desde la tendencia a naturalizar el cuidado de la niñez como responsabilidad de la mujer; lo que mantiene los escenarios de desigualdad, las formas de opresión que ocurren en la división sexual del trabajo y en el desarrollo de la labor del cuidado. Ello lleva a la reproducción de relaciones de dominación y desigualdad en las familias y la sociedad, como parte del sistema invisible de disposiciones en el que se encuentran las mujeres y las crianças.

Además, las prácticas accionadas en relación del cuidado entre mujer/ criança corresponden a escenarios de vulnerabilidad en los que convergen diversas formas de opresión. O sea, las representaciones que circulan en la práctica de cuidado dan cuenta de unas dinámicas sociales dominantes que mantienen el orden social, mostrándose como formas predominantes de generar bienestar a causa de las vulnerabilidades con las que las crianças se encuentran en dicho ámbito social, desde la perspectiva de las cuidadoras. Como consecuencia se estructuran relaciones sociales que mantienen las dinámicas de desigualdad en el territorio.

No obstante, las mujeres reconocen que es necesario ver a la niña como un sujeto fuerte y educar al niño para ser parte activa de las labores domésticas, porque de lo que se habla es del futuro y su trabajo como cuidadoras lo construye, elementos sobre los que sería importante ahondar e investigar.

Por todo ello, el cuidado como acto relacional debe ser comprendido como una actividad colectiva y no solo de la mujer/madre, lo que lleva a plantear que para esta investigación es esencial que se garantice el cuidado de la primera infancia no como un deber de la familia y menos aún como una obligación de la madre, sino como un derecho universal que debe brindar todos beneficios a la niñez y a quien desee desarrollar el trabajo del cuidado.

Por consiguiente, se deben crear políticas públicas que protejan y/o potencialicen el trabajo que realizan estas mujeres en el espacio privado. Ya que, 
consecuentemente generaría la transformación de los escenarios a los que están expuestos estos sujetos, porque modificaría las prácticas a las cuales responden las dinámicas de cuidado en este territorio, pues es claro que estas se forman en un escenario de violencia y desigualdad.

\section{Referencias}

BOGOTÁ. Rostros y rastros: razones para construir ciudad. Publicación trimestral de la dirección de equidad y políticas poblacionales de la Alcaldía mayor de Bogotá, n. 5. ene./mar., 2014. Disponible en: http://www.sdp.gov.co/sites/default/files/rostros_ rasros_5.pdf Acceso: 8 de feb. 2021.

BOGOTÁ. Primera encuesta distrital de demografía y salud Bogotá - EDDS. Alcaldía Mayor de Bogotá, 2011. Disponible en: http://www.sdp.gov.co/sites/default/files/ dice118-cartillaencuestademsaludi-2011.pdf. Acceso: 20 de feb. 2021.

BOURDIEU, Pierre. Una imagen aumentada en: La dominación masculina. Barcelona: Anagrama, 2000.

BOURDIEU, Pierre. Razões práticas sobre a teoria da ação, Trad. Mariza Corrêa. 8.ed. Campinas: Papirus, 2007.

CHICA, Jiménez F ¿Quién mata a los jóvenes en Ciudad Bolívar? El espectador, 20 sep. 2015. Disponible en: http://www.elespectador.com/noticias/bogota/quien-mata-losjovenes-ciudad-bolivar-articulo-587519 Acceso: 23 de feb. 2021.

CHODOROW, Nancy. El ejercicio de la maternidad. Barcelona: Editorial Gedisa, 1984.

FALS BORDA, Orlando. Conocimiento y poder popular. Bogotá: Editorial presencia, 1985.

HARAWAY, Donna J. Género para un diccionario marxista: la política sexual de una palabra. Madrid: Cátedra, 1995, p. 213-251. Disponible en: https://herramienta.com. ar/articulo.php?id=1780 Acceso: 15 de ene. 2021.

HIRATA, Helena. Mudanças e permanências nas desigualdades de gênero: divisão sexual do trabalho numa perspectiva comparativa. Friedrich Ebert Stiftung Brasil, n. 7. 2015. Disponible en: https://library.fes.de/pdf-files/bueros/brasilien/12133.pdf Acesso: 20 de feb. 2021.

HIRATA, Helena. Teorias e práticas do care: estado sucinto de arte, dados de pesquisa e pontos em debate. In: FARIA, Nalu; MORENO, Renata (org.). Cuidado, trabalho e 
autonomia das mulheres. São Paulo: SOF, 2010, p. 42-56. Disponible en: http://sof2. tempsite.ws/wp-content/uploads/2010/11/cuidado_trabalho_e_autonomia_das_ mulheres.pdf . Acceso: 15 de ene. 2021.

HIRATA, Helena; KERGOAT, Danièle. Novas configurações da divisão sexual do trabalho. Cadernos de Pesquisa, v. 37. n. 132. São Paulo: 2007, p. 595-6o9. Disponible en: http://scielo.br/pdf/cp/v37n132/ao537132. Acceso: 18 de ene. 2021.

KERGOAT, Danièle. Divisão sexual do trabalho e relações sociais de sexo. In: HIRATA, Helena; LABORIE, Françoise; DOARÉ, Hélène; SENOTIER, Daniele (org). Dicionário crítico do feminismo. São Paulo: Unesp, 2003, p. 67-84

MOLINIER, Pascale; PAPERMAN, Patricia. Descompartimentar a noção de cuidado? Revista Brasileira de Ciência Política, n. 18, 2015, p. 43-57. Disponible en: https://www. scielo.br/pdf/rbcpol/n18/2178-4884-rbcpol-18-ooo43.pdf. Acceso: 9 de feb. 2021.

MOLINIER, Pascale. El trabajo de cuidado y la subalternidad. HAL archives-ouvertes. Bogotá: Universidad Nacional de Colombia, 2012. Disponible en: https://hal.archivesouvertes.fr/hal-01075702/document. Acceso: 9 de feb. 2021.

QUIROGA DIAZ, Natalia. Economía del cuidado. Reflexiones para un feminismo decolonial en Abya Yala. In:ESPINOSA MIÑOSA, Yuderkys, GÓMEZCORREAL, Diana; OCHOA MUÑOZ, Karina (Ed). Tejiendo de otro modo: Feminismo, epistemología y apuestas descoloniales. Popayán: Editorial Universidad del Cauca, 2014, p. 161-175.

SALETTI CUESTA, Lorena. Propuestas teóricas feministas, en relación con el concepto de maternidad. Revista Clepsydra, n. 7, 2008, p. 169-183. Disponible en: https://riull.ull.es/xmlui/bitstream/handle/915/14275/CL_07_\%282008\%29_11. pdf? sequence=1\&isAllowed=y Acceso: 12 de feb. 2021.

SCOTT, Joan. El género: como una útil para el análisis histórico. In: LAMAS, Marta (Org.). El género: La construcción cultural de la diferencia sexual. Universidad Nacional Autónoma de México: 1996, p. 265-302. Disponible en: https://www.legisver. gob.mx/equidadNotas/publicacionLXIII/El\%2ogenero.\%2oLa\%2oconstruccion\%2o cultural\%2ode\%2ola\%2odiferencia\%2osexual.pdf . Acceso: 3 de feb. 2021.

SORJ, Bila; FONTES, Adriana. O care como regime estratificado: implicações de gênero e classe social. In: HIRATA, Helena; ARAUJO, Nadya (org.). Cuidado e cuidadoras as várias faces do trabalho do care. São Paulo: Atlas, 2012, p. 103-116.

THIRY-CHERQUES, Roberto. Pierre Bourdieu: a teoria na prática. Revista de administração pública, Rio de Janeiro, v. 40, n.1, 2006, p. 27-53. Disponible en: https:// doi.org/10.159o/So034-761220060oo100003. Acceso: 15 de ene. 2021. 
TRONTO, Joan C. "Más allá de una diferencia de género. Hacia una teoría del cuidado. In: Signs: Jornal of women in culture and society, v. 12. University of Chicago, 1987. Disponible en: http://www.unsam.edu.ar/escuelas/humanidades/centros/cedehu/ material/(13)\%20Texto\%20Joan\%2oTronto.pdf .Acceso: 22 de feb. 2021.

Recebido em 21/03/2021.

Aceito em 25/05/2021. 